\title{
Therapeutic Clinical Trial
}

National Cancer Institute

\section{Source}

National Cancer Institute. Therapeutic Clinical Trial. NCI Thesaurus. Code C39536.

A clinical study that involves administering of exposure to the agent/agents to subjects with particular disease to elucidate the most appropriate treatment for a specific medical condition, or to prolong or improve the patient's life. 